\title{
Phase Formation Modeling of an Alloy 706 Casting Using
}

\author{
Computational Thermodynamics
}

\author{
B. A. Boutwell", R. G. Thompson*, N. Saunders", S. K. Mannan", and J. J. deBarbadillo" \\ \# Inco Alloys International Inc., Huntington, WV \\ + ThermoTech Ltd., Guildford - Surrey, UK \\ * Dept. of Materials \& Mechanical Eng., University of Alabama at Birmingham, Birmingham, AL
}

\begin{abstract}
Computational thermodynamics to perform alloy and process modeling for Ni-based superalloys is a new technology which has become available to industry. This paper presents an application of the technology to a superalloy of current interest. The objective of this paper is to show how results obtained from the computational program Thermo-Calc ${ }^{{ }_{m}} 1$ using a recently developed thermodynamic database for Ni-based superalloys ${ }^{2}$ can be applied to help understand solidification in INCONEL $\left.{ }^{(}\right)$alloy 706. The ability of the computational method to predict both stable and metastable phase formation is explored.
\end{abstract}

INCONEL ${ }^{\circledR}$ alloy 706 was chosen because of its importance in the manufacture of large land-based yas turbine disks. In addition, the alloy's complex structure tested the viability of the computational method. The phases examined were: Laves, MC carbide, and the $\gamma$ matrix. Quantitative microstructure evaluation and $X$-ray dispersive analysis were performed on an as-cast, vacuum induction melted (VIM) ingot. Differential Thermal Analysis (DTA) was performed on selected samples of the alloy. The experimental data was compared to the data generated via the computational approach.

тм 'I'hermo-Calc, trade name of the Division of Computational Thermodynamics, Royal Institute of Technology, Stockholm, Sweden.

(B) INCONEL is a trademark of the INCO group of companies.

\section{Introduction}

Computational thermodynamic techniques, known as the CALPHAD (CALculation of PHAse Diagrams) method, offer a new tool for alloy design, process development, and service life evaluation. Since their initial development approximately 25 years ago, the CALPIIAD methods have developed to the point where they are now being used in the steel and aluminum industries on a regular basis. It is the flexibility of being able to calculate phase equilibria and thermodynamic properties for multicomponent alloy systems that has proven their value to the steel and aluminum industry. With the recent development of a Ni-alloy database ${ }^{2}$, the same capabilities of the CALPHAD method are being offered to the Ni-based superalloy industry. This paper will present the results of a comparison between a Scheil solidification simulation of alloy $706 \mathrm{using}$ the CALPHAD methods and experimental results from a 30 inch diameter VIM cast ingot. The purpose of this research project is to explore the capability of computational thermodynamics to simulate a non-equilibrium process, the solidification of an industrial casting.

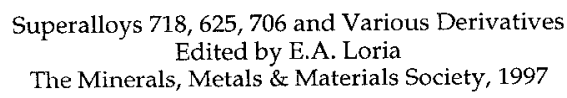




\section{Background on Computational Thermodynamics}

Computational thermodynamic techniques are based on the use of mathematical models to represent the thermodynamic properties of the system of interest. The mathematical models used to describe the properties of a system will vary depending on phase composition, phase structure, and arrangement/bonding of elements within each phase. The general representation of all models can be represented by the equation:

$$
\Delta G=\Delta G^{\circ}+\Delta G_{m i x}^{\text {ideal }}+\Delta G_{\text {mix }}^{\text {excess }}
$$

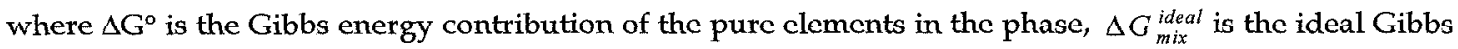
energy of mixing, and $\Delta G_{\text {mix }}^{\text {excess }}$ is the excess Gibbs energy of mixing term. The general expressions for $\Delta G^{\circ}$ and $\Delta G_{m i x}^{\text {ideal }}$ are given by the equations:

$$
\begin{aligned}
& \Delta G^{\circ}=x_{i} \sum_{i} \Delta G_{i}^{\circ} \\
& \Delta G_{\text {mix }}^{\text {ideal }}=R T \sum_{i} x_{i} \ln x_{i}
\end{aligned}
$$

where $x_{i}$ is the mole fraction of component $i, \mathrm{R}$ is the gas constant, and $\mathrm{T}$ is temperature. The excess Gibbs energy is generally the most difficult parameter to model.

Though there are various models used to describe the excess Gibbs energy, all of the models require an input of coefficients to uniquely describe the properties of a phase for a given alloy system. These coefficients are stored in databases which are available either in open literature or are proprietary ${ }^{2,3}$. Once the appropriate models are in place, the necessary coefficients to describe a specific phase are taken from the database and substituted into the model. The calculation of $\Delta G$ for the phase is then a straightforward procedure, though quite tedious, which is best relegated to a computer.

The Ni-alloy database used in the present work can model multi-component alloys with the following elements:

\section{$\mathrm{Ni}-\mathrm{Al}-\mathrm{Co}-\mathrm{Cr}-\mathrm{Fe}-\mathrm{Mo}-\mathrm{Nb}-\mathrm{Ti}-\mathrm{Zr}-\mathrm{B}-\mathrm{C}$}

The computational thermodynamic software Thermo-Calc ${ }^{\mathrm{Tm}} 1$ utilizes a Gibbs free energy minimization procedure to calculate the thermodynamic properties and phase equilibria of the chosen system. For a given set of conditions, the computer determines the free energy change for each possible combination of phases and phase compositions and selects the state that minimizes the overall Gibbs energy of the system.

While thermodynamics deals with equilibrium states and cannot describe kinetic effects, the CALPHAD method is flexible enough to incorporate data on metastable phases and can handle calculations on metastable equilibrium. The software can be configured to eliminate certain phases from its calculations and then calculate the next lowest Gibbs energy state. This is quite useful in modeling some limited types of kinetic phenomenon as one is not restricted to modeling only stable phases but can evaluate metastable states in an alloy.

INCONEL $^{\circledR}$ alloy 706

INCONEL $^{\circledR}$ alloy 706 is a Ni-Fe based superalloy which is used in wrought form in large power generation turbine engines. Inco Alloys International developed alloy 706 in the late sixties with specific goals in mind: (1) an alloy which could be cast with fewer segregation defects in larger ingot sizes than alloy $718,(2)$ an alloy which could be forged with current forging capacities in the larger ingot sizes, (3) an alloy with better machinability than alloy 718 , and (4) an alloy which is more economical than alloy 
$718^{4,5}$. The chemistry of alloy 706 was derived from alloy 718 to meet these metallurgical objectives. The nominal composition of alloy 718 and alloy 706 are shown in Table $\mathrm{I}$.

Table I: Nominal composition of Inconel alloys 718 and 706.

\begin{tabular}{|c|c|c|c|c|c|c|c|c|c|c|c|}
\hline Alloy & $\mathrm{Ni}$ & $\mathrm{Cr}$ & $\mathrm{Fe}$ & $\mathrm{Mo}$ & $\mathrm{Nb}$ & $\mathrm{Ti}$ & $\mathrm{Al}$ & $\mathrm{C}$ & $\mathrm{B}$ & $\mathrm{Mn}$ & $\mathrm{Si}$ \\
\hline 718 & 53.5 & 19.0 & Balance & 3.05 & 5.3 & 0.9 & 0.5 & 0.03 & 0.002 & 0.10 & 0.10 \\
\hline 706 & 41.5 & 16.0 & Balance & - & 2.9 & 1.75 & 0.2 & 0.02 & 0.004 & 0.10 & 0.10 \\
\hline
\end{tabular}

Alloy 706 is a precipitation strengthened alloy with both $\gamma^{\prime}, \mathrm{FCC}^{\prime} \mathrm{Ni}\left(\mathrm{Al}, \mathrm{I}^{\mathrm{i}} \mathrm{i}\right)$, and $\gamma^{\prime \prime}, \mathrm{BCT} \mathrm{Ni}_{3} \mathrm{Nb}$, precipitates. The $\gamma^{\prime \prime}$ precipitate is the more numerous phase ${ }^{6}$. In the as-cast material MC carbide$(\mathrm{Nb}, \mathrm{Ti}) \mathrm{C}$, Laves- IICP ( $\mathrm{Fe}, \mathrm{Ni})_{2} \mathrm{Nb}$, and Eta phase- HCP $\mathrm{Ni}_{3} \mathrm{Ti}^{1,5}$ could be present. The Laves phase is detrimental to mechanical properties and is eliminated during subsequent heat treating and forging operations after casting. Eta phase formation has shown to improve stress rupture properties of alloy $706^{7,8}$ and may be desirable in the final microstructure depending on the mechanical properties desired. These points must be considered when preparing an alloy 706 ingot for hot working. A knowledge of the initial microstructure is desirable in designing the forging/heat treating process to avoid any potential pitfalls during processing and this is where computational thermodynamics can potentially be utilized to provide answers or warnings about a processing treatment.

\section{Experimental Procedure}

Two sections from a 30-inch diameter VIM ingot were taken from the head end. The composition of the ingot is given in Table II. One section was taken near the center of the ingot while the other was taken near the edge of the ingot. The samples were polished and chemically etched using a swab etchant $\left(92 \mathrm{ml} \mathrm{HCl}_{5} 5 \mathrm{ml} \mathrm{H}_{2} \mathrm{SO}_{4}, 3 \mathrm{ml} \mathrm{HNO}_{3}\right.$ ) to reveal Laves, carbide, and $\eta$ phases. Stereological measurements of volume fractions of carbide and Laves were performed using an optical microscope at 500x magnification with a 64-point grid.

Table II: Alloy 706 VIM ingot composition in weight percent.

\begin{tabular}{|c|c|c|c|c|c|c|}
\hline $\mathrm{Ni}$ & $\mathrm{Cr}$ & $\mathrm{Fe}$ & $\mathrm{Nb}$ & $\mathrm{Ti}$ & $\mathrm{Al}$ & $\mathrm{C}$ \\
\hline $\mathbf{4 1 . 4 6}$ & $\mathbf{1 6 . 1 7}$ & 36.94 & 3.01 & 1.82 & 0.27 & 0.02 \\
\hline
\end{tabular}

Differential Thermal Analysis (DTA) was performed on samples from the center and edge sections. The thermal cycle used an initial heating rate of $100^{\circ} \mathrm{C} / \mathrm{min}$ to $900^{\circ} \mathrm{C}$ and then a heating rate of $20^{\circ} \mathrm{C} / \mathrm{min}$ to $1420{ }^{\circ} \mathrm{C}$. The samples were then isothermally held at $1420{ }^{\circ} \mathrm{C}$ for 5 minutes to reach thermal equilibrium prior to cooling at a rate of $20{ }^{\circ} \mathrm{C} / \mathrm{min}$ to $900{ }^{\circ} \mathrm{C}$. The sample was then cooled at the maximum rate attainable by the DTA system until room temperature. The transformation temperatures were located by determining the temperatures at which the $\Lambda \mathrm{T}$ curve deviated from the local baseline.

Samples of the center and edge sections were also examined in a Scanning Electron Microscope (SEM) using secondary electron imaging, back-scattered electron imaging, and energy dispersive spectroscopy (EDS). Several qualitative EDS spectra and semi-quantitative EDS compositions of the gamma matrix, Laves phase, and carbide phase were collected for both center and edge pieces. The SEM analysis was necessary to provide information on phase composition. Back-scattered electron imaging also provided some information as to the nature of the composition of Eta phase since the needle-like precipitates were too small to obtain an EDS spectra.

\section{Results and Discussion}

\section{$\underline{\text { Scheil solidification simulation }}$}

The Scheil solidification simulation technique utilizing computational thermodynamics can be illustrated by examining a section of a hypothetical binary phase diagram as shown in Figure 1. The calculations begin at a temperature above the liquidus temperature of an alloy of composition $C_{0}$. The alloy begins to cool and at a temperature, $T_{L}$, the first solid begins to form with a composition of $C_{S 1}$ and a liquid composition of $\mathrm{C}_{\mathrm{L} 1}$. Since only a small fraction of solid forms, the composition of the liquid is not drastically altered from the bulk alloy composition. At the next temperature step $T_{2}$, more solid has 


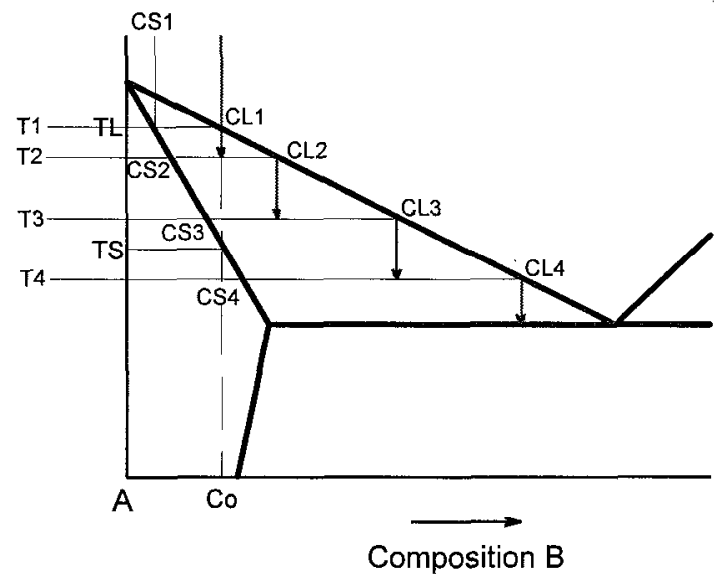

Figure 1: Binary System Schematic of the Scheil Simulation Methodology

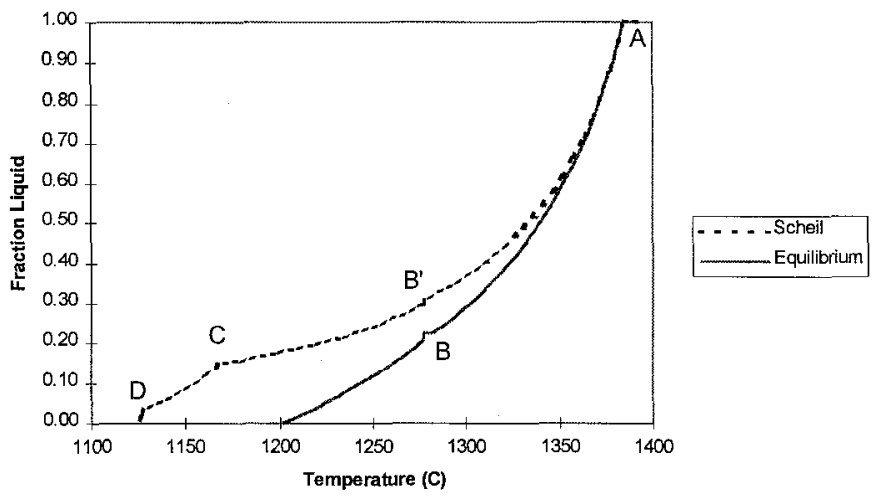

Figure 2: Fraction Liquid vs. Temperature Curves for Equilibrium and Scheil Computer Simulations.

formed with a new composition of $C_{52}$ and a new liquid composition of $C_{L 2}$. At $T_{2}$, the overall liquid composition is changed from $C_{O}$ to $C_{L_{2}}$ to approximate solute enrichment of the liquid due to segregation during solidification. The same procedure is repeated for temperature $\mathrm{T}_{3}$ and all the following temperature steps until the liquid phase becomes unstable and complete solidification occurs. While the illustration describes a binary system, the same procedure can be performed for a multicomponent system using computational thermodynamics software.

Plots of fraction liquid versus temperature calculated for the VIM ingot composition utilizing both equilibrium and Scheil simulations are shown in Figure 2. For equilibrium solidification, the only phases that were calculated to solidify from the liquid were the Gamma matrix with solidification beginning at $1385.0^{\circ} \mathrm{C}$ (pt. A) and the MC carbide (pt. B) which began to solidify at $1282.6^{\circ} \mathrm{C}$. The alloy completely solidified at $1203.0^{\circ} \mathrm{C}$ giving a solidification range of $182.0^{\circ} \mathrm{C}$. Using the Scheil simulation, solidification begins at $1385.0^{\circ} \mathrm{C}$ with the Gamma matrix (pt. A as in the equilibrium simulation) with the MC carbide precipitating at a slightly lower temperature of $1277.4^{\circ} \mathrm{C}\left(\mathrm{pt} . \mathrm{B}^{\prime}\right)$. This is then followed by Laves phase at $1167.6^{\circ} \mathrm{C}$ (pt. C), and finally Fta forming at $1126.4^{\circ} \mathrm{C}$ (pt. D). Complete solidification occurs at 1125.1 ${ }^{\circ} \mathrm{C}$ giving a solidification range of $259.9^{\circ} \mathrm{C}$. For both the equilibrium and Scheil simulations, the curves show changes in slope when a new phase begins to precipitate from the liquid. 


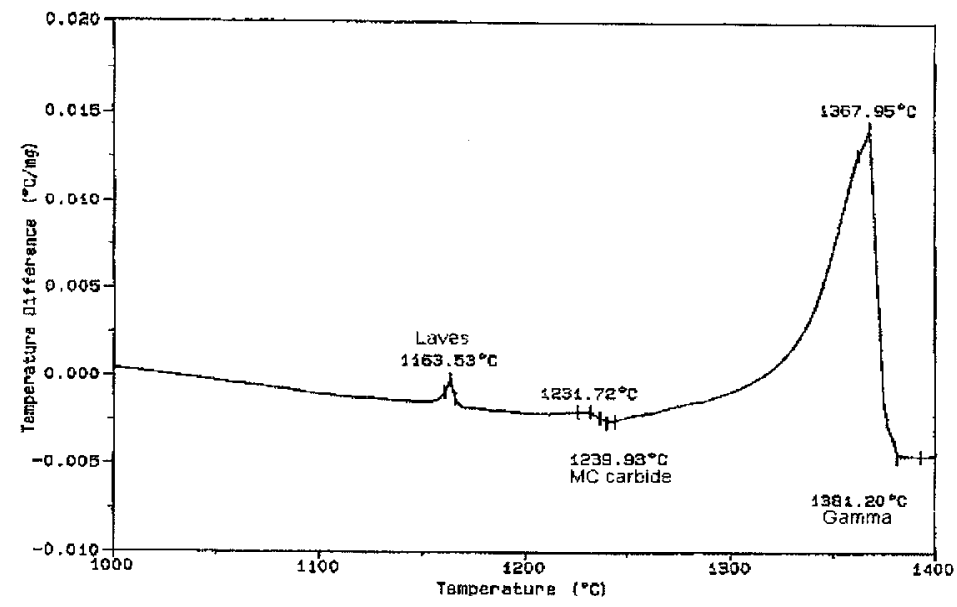

(a)

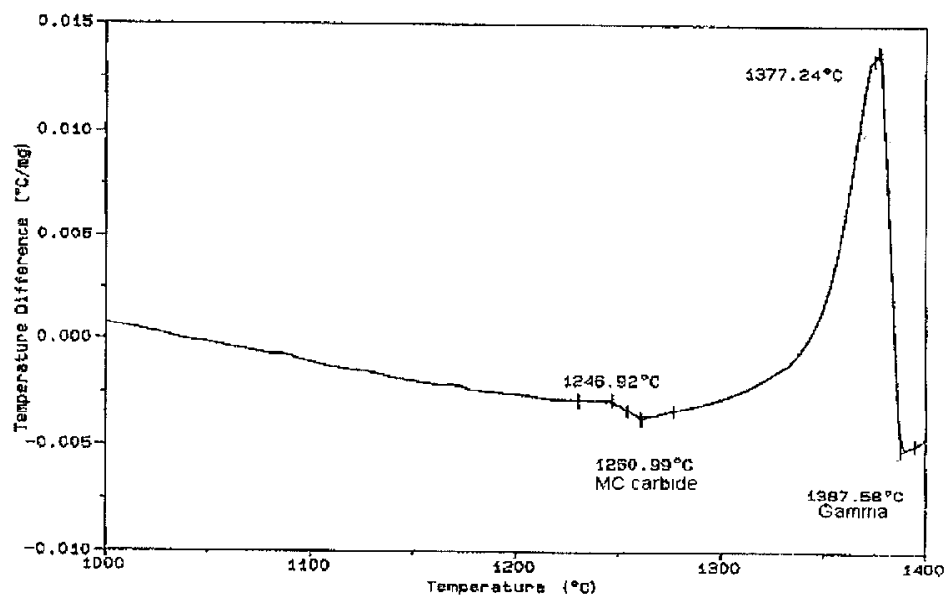

(b)

Figure 3: DTA curves for (a) center section and (b) edge section on cooling

The results from the DTA analysis of the center and edge pieces are shown in Figure 3. The DTA results are summarized in Table III along with the CALPHAD simulation data. There is good agreement between the measured onset temperatures for the $\gamma$ liquidus and the Laves start temperatures; however, the MC carbide phase begins to precipitate at temperatures 17 to $38{ }^{\circ} \mathrm{C}$ lower than predicted by the Scheil simulation.

Table III: DTA and Scheil Simulation Results

\begin{tabular}{|l|c|c|c|c|c|}
\hline & $\gamma$ Liquidus & MC start & Laves start & Eta start & $\gamma$ solidus \\
\hline Center (DTA) & $1381^{\circ} \mathrm{C}$ & $1240^{\circ} \mathrm{C}$ & $1164^{\circ} \mathrm{C}$ & -- & -- \\
\hline Edge (DTA) & $1388^{\circ} \mathrm{C}$ & $1261^{\circ} \mathrm{C}$ & - & -- & -- \\
\hline Equilibrium Simulation & $1385^{\circ} \mathrm{C}$ & $1283^{\circ} \mathrm{C}$ & -- & -- & $1203^{\circ} \mathrm{C}$ \\
\hline Scheil Simulation & $1385^{\circ} \mathrm{C}$ & $1277^{\circ} \mathrm{C}$ & $1168^{\circ} \mathrm{C}$ & $1126^{\circ} \mathrm{C}$ & $1125^{\circ} \mathrm{C}$ \\
\hline
\end{tabular}




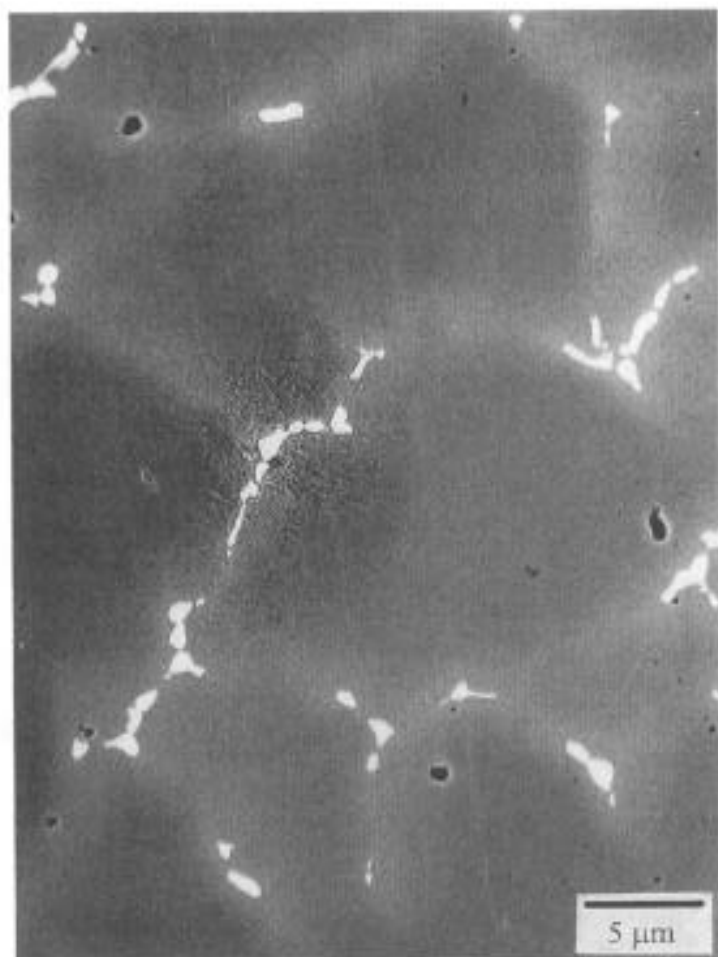

(a)

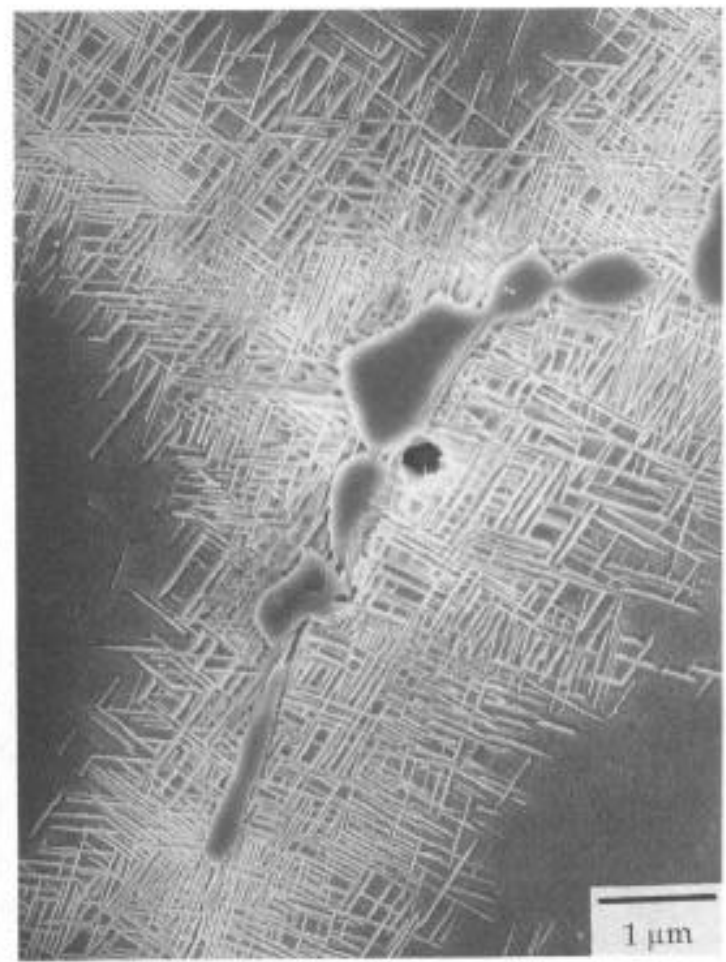

(b)

Figure 4: Selected SEM photomicrographs

(a) Back-scattered electron image showing general microstructural features,

(b) Secondary electron image close-up of Laves phase surrounded by Eta needles

\section{Phase Fractions}

Representative microstructures for both sections of the casting are shown in Figure 4. The stereological measurement of phase fractions of MC carbide and Laves phase are summarized in Table IV along with the simulation results. The calculated phase fractions are in good agreement with the experimentally measured values, especially the measurement for carbide volume fraction. The simulation results for the Laves phase, while not as good as the carbide phase, still provide a good approximation of the measured results. The simulation also predicted that a very small amount of Eta phase would form from the last remaining liquid. This quantity is probably too small to measure stereologically and is masked by the solid state Widmanstatten precipitation of this phase during cooling.

Table IV: Experimental and Simulated $V_{v}$ Values for Carbide and Laves Phase.

\begin{tabular}{|l|c|c|c|c|}
\hline \multirow{2}{*}{} & \multicolumn{2}{|c|}{ Carbide } & \multicolumn{2}{c|}{ Laves } \\
\cline { 2 - 5 } & $\begin{array}{c}\text { Volume } \\
\text { Fraction }\left(\mathrm{V}_{\mathrm{v}}\right) \%\end{array}$ & $\begin{array}{c}\text { Coefficient of } \\
\text { Variation for } \mathrm{V}_{\mathrm{v}}\end{array}$ & $\begin{array}{c}\text { Volume Fraction } \\
\left(\mathrm{V}_{\mathrm{v}}\right) \%\end{array}$ & $\begin{array}{c}\text { Coefficient of } \\
\text { Variation for } \mathrm{V}_{v}\end{array}$ \\
\hline Center section & $0.12 \pm .0195$ & 0.08 & $1.2 \pm 0.14$ & 0.06 \\
\hline Edge section & $0.14 \pm 0.040$ & 0.15 & $1.3 \pm 0.17$ & 0.06 \\
\hline Scheil Simulation & 0.16 & - & 4.0 & - \\
\hline
\end{tabular}

\section{Phase Compositions}

In addition to phase fractions, the Scheil simulation can also predict the composition change of a phase with temperature and Figure 5 shows these results for the Gamma, Laves, and MC carbide phases. For comparison, semi-quantitative EDS analysis results are summarized in Table 5 . The EDS results are 


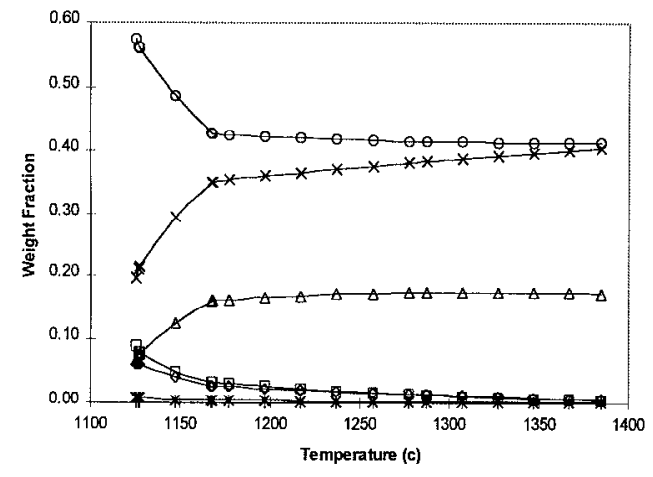

(a)

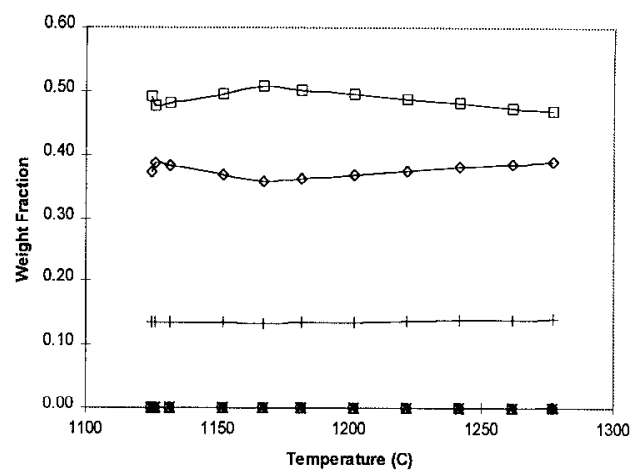

(b)

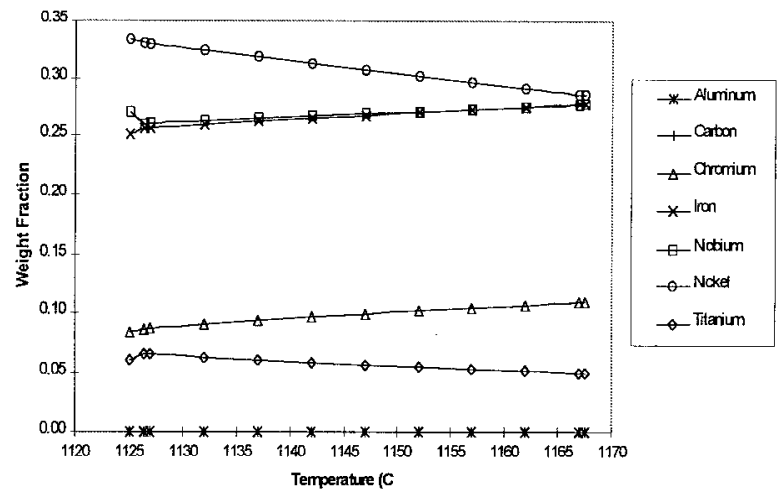

(c)

Figure 5: Calculated Composition versus Temperature Profiles for (a) Gamma, (b) MC Carbide, and (c) Laves phase.

representative of the phase composition of both the center and edge sections and represent the room temperature phase composition of a solidified casting. As Table V illustrates, the composition of theMC carbide was observed to fall within three populations based on the $\mathrm{Nb} / \mathrm{Ti}$ ratio. The most commonly observed composition was the high $\mathrm{Nb} / \mathrm{Ti}$ ratio Carbide followed by the medium $\mathrm{Nb} / \mathrm{Ti}$ Carbide with the low $\mathrm{Nb} / \mathrm{Ti}$ Carbide being the least often observed. The results of the computational simulation are comparable to the phase compositions measured by EDS for the Gamma, Laves, and the medium $\mathrm{Nb} / \mathrm{Ti}$ ratio MC carbide. The computer simulation failed to predict the presence of the low and high $\mathrm{Nb} / \mathrm{Ti}$ ratio MC carbides. Thus the CALPHAD approach can provide a good initial estimate of phase composition for $\gamma$, Laves, and the medium $\mathrm{Nb} /$ Ti ratio Carbide.

The phase compostion of Eta was also calculated but no EDS spectra could be obtained due to the fine nature of its needle like structure. Back scattered electron imaging of the Eta phase indicated that it was high in lower atomic number elements such as Ti than the $\gamma$ matrix, Laves, and MC carbide. This is in agreement with the simulation composition for Eta phase which predicted the phase would be Ti-rich and have almost no high atomic number elements present.

Table V. Semi-Quantitative X-ray Analysis Results of Phase Composition (wt.\%)

\begin{tabular}{|c|c|c|c|c|c|c|}
\hline Phase & $\mathrm{Ni}$ & $\mathrm{Cr}$ & $\mathrm{Fe}$ & $\mathrm{Nb}$ & $\mathrm{Ti}$ & $\mathrm{Al}$ \\
\hline Gamma & 39.85 & $\mathbf{1 7 . 8 2}$ & 40.23 & 0.67 & 1.43 & 0 \\
\hline $\mathrm{MC}$ (hi Nb/Ti) & $\mathbf{1 . 8 2}$ & 0.53 & 1.25 & 78.81 & 17.58 & 0.01 \\
\hline $\mathrm{MC}$ (med Nb/Ti) & $\mathbf{1 . 5 6}$ & 0.58 & 1.23 & 59.33 & 37.26 & 0.04 \\
\hline $\mathrm{MC}$ (low Nb/Ti) & 9.86 & 4.69 & 9.37 & 3.30 & 72.79 & 0 \\
\hline Laves & 28.40 & $\mathbf{1 0 . 1 4}$ & 27.07 & 31.02 & 3.34 & 0.03 \\
\hline
\end{tabular}




\section{Simulated versus Experimental Data:}

The results clearly show that the formation of various phases in an alloy 706 casting can be predicted using computational thermodynamic modeling. Phase characteristics such as onset temperatures, composition, and volume fraction can be predicted quite accurately. The difference in measured and simulated onset temperatures can be partially attributed to the presence of trace elements $(\mathrm{S}, \mathrm{P}, \mathrm{B})$ in the alloy which tend to lower onset temperatures for MC carbide. The exact stoichiometry could also vary from simulated and semi-quantitatively measured EDS values which would affect the onset temperatures and volume fractions of the phases. In addition, the absence of trace clements such as S, P, and $\mathrm{N}$ in the Ni-alloy database also contribute to differences between simulated and experimental results.

Computational thermodynamics uses binary and ternary thermodynamic data to calculate phase equilibria for multi-component systems. 'The models used allow the CALPHAD approach to make assumptions about atomic interactions in higher order systems from the binary and ternary data. The computational thermodynamic approach is applied to a particular size ingot of unknown cooling rate to predict accurate phase formation temperatures, compositions, and volume fractions. Good agreement exists between simulated and experimental data in view of all the factors mentioned previnusly. This reveals that the predictive capability of computational thermodynamics is excellent.

\section{Conclusions}

1. The computational thermodynamic Scheil simulation of solidification generally provided very good predictions for the solidification behavior of alloy 706 .

2. The solidification start temperatures of the Gamma and Laves phase as measured by DTA analysis were predicted very well but Carbide solidification was predicted to start at somewhat higher temperatures than measured experimentally.

3. The CALHPAD Scheil simulation provided a close approximation of the volume fractions for Carbide and Laves phases in alloy 706. The volume fraction predicted for the MC carbide was closer to measured volume fractions than was the Laves prediction.

1. The compositional data obtained by EDS analysis of the alloy 706 sections correlated very well with the predicted composition versus temperature profiles for Gamma, Laves, and the medium $\mathrm{Nb} / \mathrm{Ti}$ ration MC carbide.

5. The CALPHAD simulation failed to predict the appearance of a high and low $\mathrm{Nb} / \mathrm{Ti}$ ratio $\mathrm{MC}$ carbide in the casting.

\section{References}

1. B. Sundman, User Aspects of Phase Diagrams (I.nndon, U.K. : Institue of Metals, 1991), ed. F. H. Hayes, 130

2. N. Saunders, "Phase Diagram Calculations for Ni-Based Superalloys," Superalloys 1996 (Warrendale, PA : The Metallurgical Society, 1996), 101-110.

3. N. Saunders, "Phase Diagram Calculations for High Temperature Structural Materials" (Paper presented at the Royal Sociely Meeling on High-Temperature Structural Materials, London, U.K., 23 November, 1994),

4. H. T. Fiselstein, Properties of Inconel Alloy 706, Materials Engineering Congress, Cleveland, OH., 1970.

5. P. W. Schilke, J. J. Pepe, and R. C. Schwant, "Alloy 706 Metallurgy and Turbine Wheel Application," Superalloys 718, 625, 706, and Various Derivatives (Warrendale, PA: The Metallurgical Society, 1994), ed. E. A. Loria, 1-11.

6. L. R-my, J. Laniesse, and H. Aubert, "Precipitation Behavior and Creep Rupture of 706 Type Alloys," Materials Science and Engineering, 38 (1979), 227-239.

7. J. H. Moll, G. N. Maniar, and D. R. Muzyka, "The Microstructure of 706, a New Fe-Ni-Base Superalloy," Metallurgical Transactions, 2 (August, 1971), 2143-2151.

8. J. H. Moll, G. N. Maniar, and D. R. Muzyka, "Heat Treatment of 706 Alloy for Optimum $1200^{\circ} \mathrm{F}$ Stress-Rupture Properties," Metallurgical Transactions, 2 (August, 1971), 2153-2160. 